\title{
Feasibility and Safety of Neoadjuvant Alectinib in Pulmonary Invasive Mucinous Adenocarcinoma with ALK Rearrangement: Case Report and Literature Review
}

\author{
Rumeng Gul,2 \\ Ziling Shi ${ }^{1,2}$ \\ Ting Duan' \\ Meijun Song' \\ 'Department of Respiratory Medicine, \\ Zhejiang Provincial People's Hospital, \\ Affiliated People's Hospital, Hangzhou \\ Medical College, Hangzhou, Zhejiang, \\ People's Republic of China; ${ }^{2}$ Graduate \\ School of Clinical Medicine, Bengbu \\ Medical College, Bengbu, People's \\ Republic of China
}

\begin{abstract}
Background: Pulmonary invasive mucinous adenocarcinoma (IMA) is a rare variant of lung adenocarcinoma that rarely shows anaplastic lymphoma kinase (ALK) rearrangement. Alectinib (tyrosine kinase inhibitors) has been listed as category 1 recommendations for advanced ALK + NSCLC first-line therapy due to low toxicity and excellent efficacy, and its median progression-free survival is 34.8 months. Here, we report a case of a patient with ALK-rearranged lung IMA who showed favorable results to neoadjuvant alectinib.

Case: A 67-year-old man with no history of smoking was diagnosed with clinical stage as IIIB invasive mucinous adenocarcinoma based on clinical symptoms, chest CT and pathological findings. The anaplastic lymphoma kinase (ALK) fusion status was assessed by realtime PCR. After acquiring informed consent from the patient, we offered neoadjuvant alectinib at a dosage of $150 \mathrm{mg}$ twice per day for three cycles (84 days), all lesions were undetectable on chest CT. Later, a thoracoscopic left lobectomy was performed. The postoperative pathological showed that a small amount of tumor cells remained, and the TNM stage was downstaged as T1aN0M0 IA.

Conclusion: To our knowledge, this is the first case discussing the treatment of ALKrearranged IMA of the lung with neoadjuvant alectinib. Alectinib is an effective ALK inhibitor, and in cases of lung adenocarcinoma with ALK rearrangement, alectinib treatment is a reasonable and priority option. Neoadjuvant alectinib may be clinically feasible and well tolerated in locally advanced NSCLC.
\end{abstract}

Keywords: neoadjuvant alectinib, IMA, ALK rearrangement

\section{Introduction}

Pulmonary invasive mucinous adenocarcinoma (IMA) is a rare adenocarcinoma variant of lung according to the new international multidisciplinary classification system (IASLC/ATS /ERS). ${ }^{1}$ This new category of adenocarcinoma, accounting for only about $5 \%$ of lung adenocarcinomas. ${ }^{2}$ Its histology is typified by goblet or columnar tumor cells with abundant mucin. ${ }^{3}$ In addition to pathology, IMA has unique clinical, imaging and genetic characteristics, KRAS mutation has been reported to be the most common alteration in IMA, while ALK rearrangement is rare. ${ }^{4}$ We here report a case of ALK-rearranged in IMA of lung.

Anaplastic lymphoma kinase (ALK) rearrangement occurs in approximately 5\% of non-small cell lung cancer (NSCLC). ${ }^{5}$ The echinoderm microtubule-associated 
protein-like 4 (EML4)-ALK fusion formed by the inversion in chromosome 2 is the most common type in ALK rearrangement. ${ }^{6,7}$ Currently, ALK rearrangement is significant molecular target for NSCLC treatment, alectinib is an effective and highly selective second-generation ALK inhibitor. ${ }^{8}$ Unlike crizotinib (first-generation ALK inhibitor), alectinib has shown good efficacy against CNS metastases in multiple studies. ${ }^{9-11}$ According to the updated NCCN Guidelines Insights and CSCO Guidelines for Primary Lung Cancer, alectinib has been included as a Category 1 recommendation for the first-line treatment of advanced ALK + NSCLC. ${ }^{12}$ Based on the Global Phase III ALEX study (BO28984, NCT02075840), the median PFS time of alectinib was 34.8 months, which was significantly longer than that of crizotinib (10.9 months).${ }^{13}$ Moreover, compared with crizotinib, alectinib can significantly improve intracranial objective response rate (ORR) ${ }^{14}$ Alectinib is becoming increasingly important because of low toxicity and excellent efficacy. Based on previous studies, compared with surgery alone, neoadjuvant therapy increased the 5 -year recurrence-free survival rate of operable locally advanced NSCLC by $6 \% .{ }^{15,16}$ At present, there have been case reports of alectinib as neoadjuvant treatment, and the safety and feasibility of the treatment have been confirmed. ${ }^{17,18}$ However, the efficacy of alectinib as neoadjuvant treatment in pulmonary IMA has not been reported. Here, we report the excellent therapeutic effect of neoadjuvant alectinib in IMA of lung with ALK rearrangement.

\section{Case Report}

The patient, a 67-year-old man with no smoking history, was referred to the hospital for hoarseness that lasted for 20 days. Laryngoscopy revealed complete paralysis of the left vocal cord. Computed tomography (CT) of the chest showed a lobulated mass of $3.2 \times 2.4 \mathrm{~cm}$ under the pleura in the left upper lung, with mediastinal and left hilar lymphadenopathy (Figure 1A). A CT-guided percutaneous lung biopsy of the lung lesion was performed, and a diagnosis of invasive mucinous adenocarcinoma (IMA) of lung was made by pathologists (Figure 2A and B), a lot of mucus fills the alveolar space. In addition, brain magnetic resonance imaging and whole-body bone imaging and PET-CT scan revealed no distant metastasis. And laryngoscopy revealed that the left vocal cord was completely paralyzed, considering that the lesion invaded the recurrent laryngeal nerve. A stage IIIB (T4N2M0) IMA of lung was confirmed by histology and imaging.
Then, the biopsied tissue was examined for EGFR, ALK and ROS1 gene panels by real-time fluorescence polymerase chain reaction (real-time PCR), and finally the EML4-ALK rearrangement was detected. Subsequently, the patient began to receive treatment with alectinib (150 mg twice daily), a selective second-generation ALK inhibitor. After the first cycle of treatment, the patient's hoarseness symptoms improved significantly. CT of the chest showed that the size of the left upper lobe lobulated mass was $1.7 \times 1.4 \mathrm{~cm}$, which was significantly smaller than before (Figure 1B). After the second cycle of treatment, the chest $\mathrm{CT}$ showed that the tumor lesions were further reduced (Figure 1C). After three cycles (84 days) of neoadjuvant treatment, no solid lesions were found on chest $\mathrm{CT}$, the tumor achieved complete remission (CR) (Figure 1D), and no adverse drug reactions were seen. Subsequently, the patient was transferred to cardiothoracic surgery for thoracoscopic left lobectomy and systemic lymphadenectomy. Postoperative pathology revealed a small amount of scattered high-grade atypical glands in the interstitium, and fibrous tissue proliferation, foam cell aggregation, lymphocyte infiltration, multinucleated giant cell reaction, necrotic hemicalcium can be seen in the remaining lung tissue (Figure 3A and B). Then, Integrated the TNM stage was downstaged as T1aN0M0 IA. And the immunohistochemistry revealed that Ki61, cytokeratin 7 (CK7) and thyroid transcription factor-1 (TTF-1) were positive (Figure 4).

\section{Discussion}

This is the first case discussing the treatment of ALKrearranged IMA of the lung with alectinib, as far as I know. The diagnosis of lung IMA was confirmed by pathological examination, and the histological appearance is highly specific to diagnose IMA. Generally speaking, the histological appearance of IMA often show that tumor cells containing goblet and/or columnar components are abundant intracellular or extracellular mucus. According to the difference of histology, we divide IMA into two groups: mucous extracellular adenocarcinoma (MOCA) and mucous intracellular adenocarcinoma (MICA). Compared with the MOCA group, the recurrence-free survival (RFS) and overall survival (OS) of the MICA group were significantly longer, and the incidence of lymph node metastasis in MOCA group was significantly higher. ${ }^{19}$ Our patient's pathology showed a lot of mucus filling the alveolar space, which is MOCA type. On the basis of high-resolution CT (HRCT) examination, IMA is divided into the following three types: solid type, bubbling 


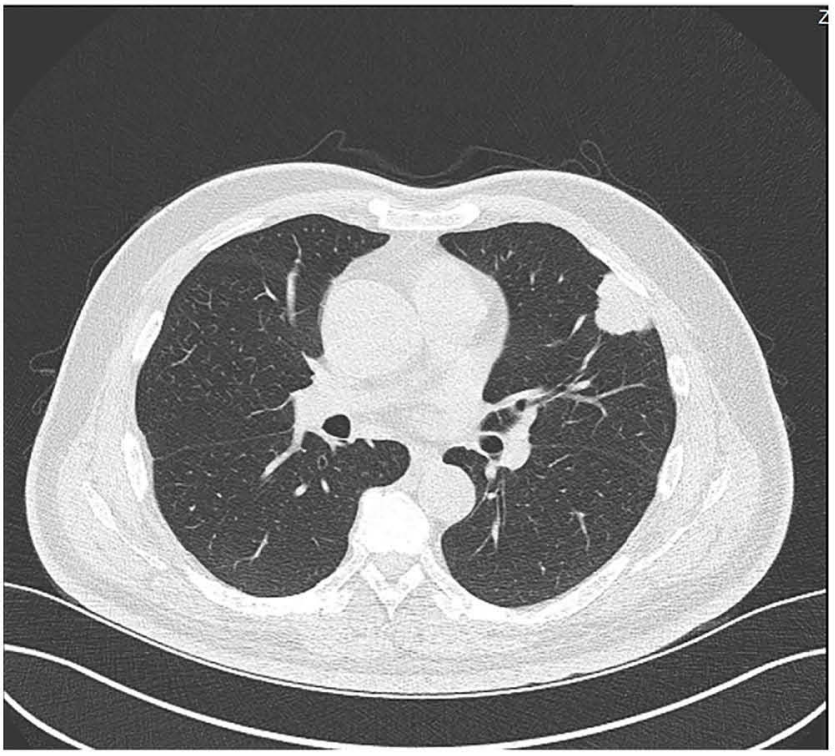

A

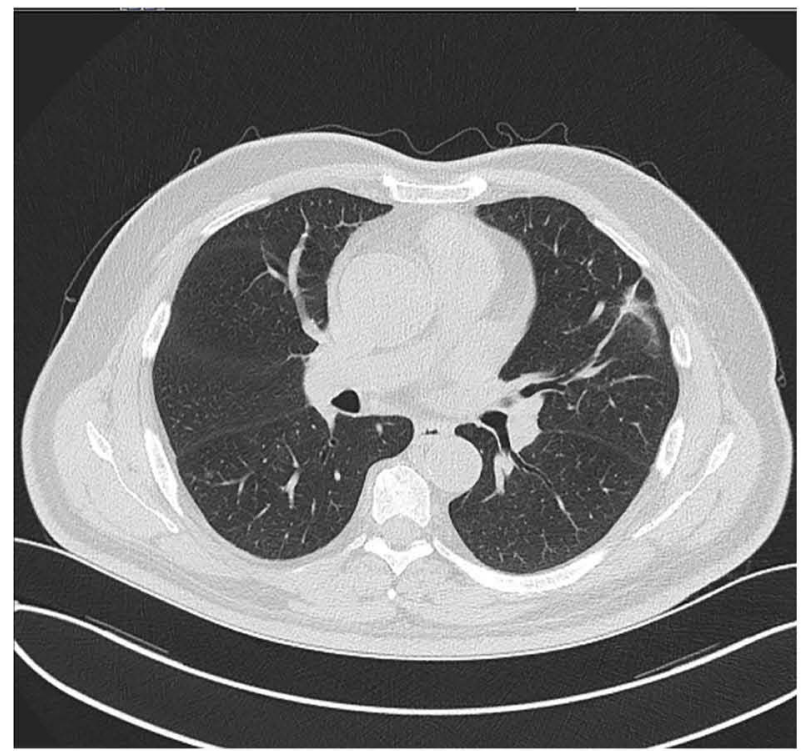

C

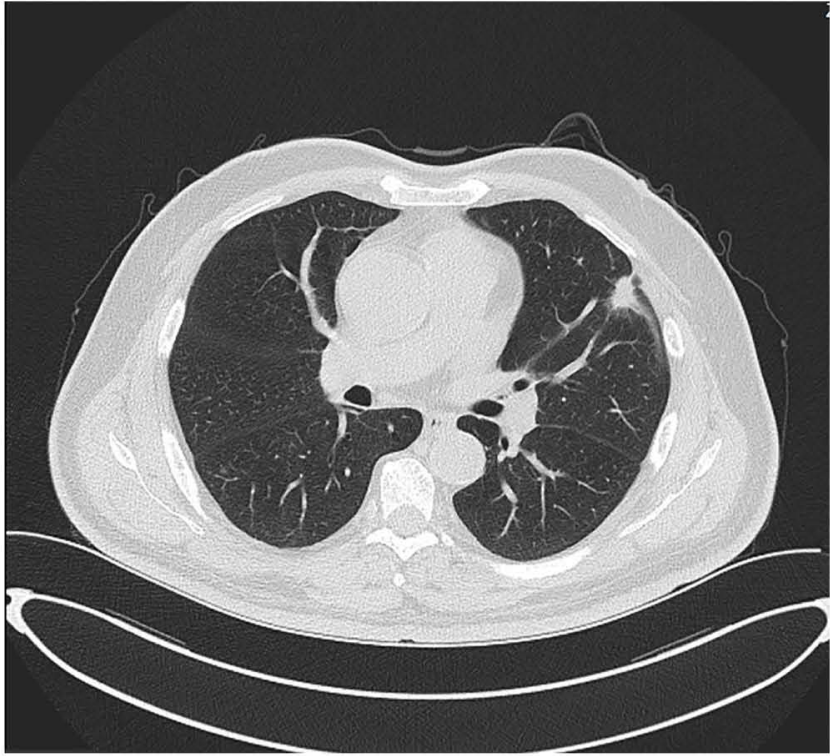

B

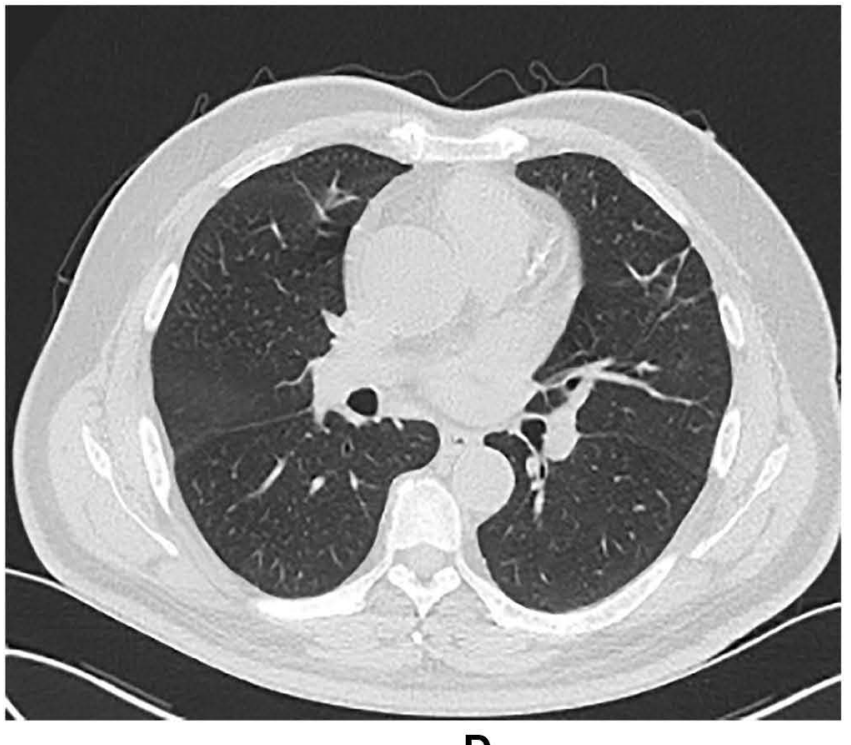

D

Figure I Computed tomography findings before and after treatment with alectinib. A computed tomography scan before treatment revealed a solitary tumor in left upper lung (A). A computed tomography scan I months after commencing treatment with alectinib revealed that dramatic reduction in tumor size (B and C), almost no presence of tumor lesion (D).

type and pneumonic type. In the case we reported, the chest CT of IMA showed an isolated mass in the left upper lung, belonging to solid type. A study suggests that the 5-year RFS rate of IMA solid type was $87.5 \%{ }^{20}$ According to a retrospective study, the expression rate of TTF1 in lung IMA immunohistochemistry was $62.1 \%$, and the expression rate of CK7 was $100 \%$, which means that all tumor cells were identified as positive for CK $7 .{ }^{21}$ In our case, both CK7 and TTF-1 test results were positive, which was consistent with this study.
Lung IMA harboring ALK rearrangement is extremely rare. A study by Kim et al showed that in 293 lung adenocarcinoma patients with ALK rearrangement, IMA accounted for $3.4 \%{ }^{22}$ ALK is a transmembrane protein receptor, and its gene is responsible for encoding a receptor tyrosine kinase called ALK, which is a member of the receptor tyrosine kinase family. ALK rearrangements create an oncogenic ALK tyrosine kinase that activates many downstream signaling pathways resulting in increased cell proliferation and survival. ${ }^{23}$ There are 


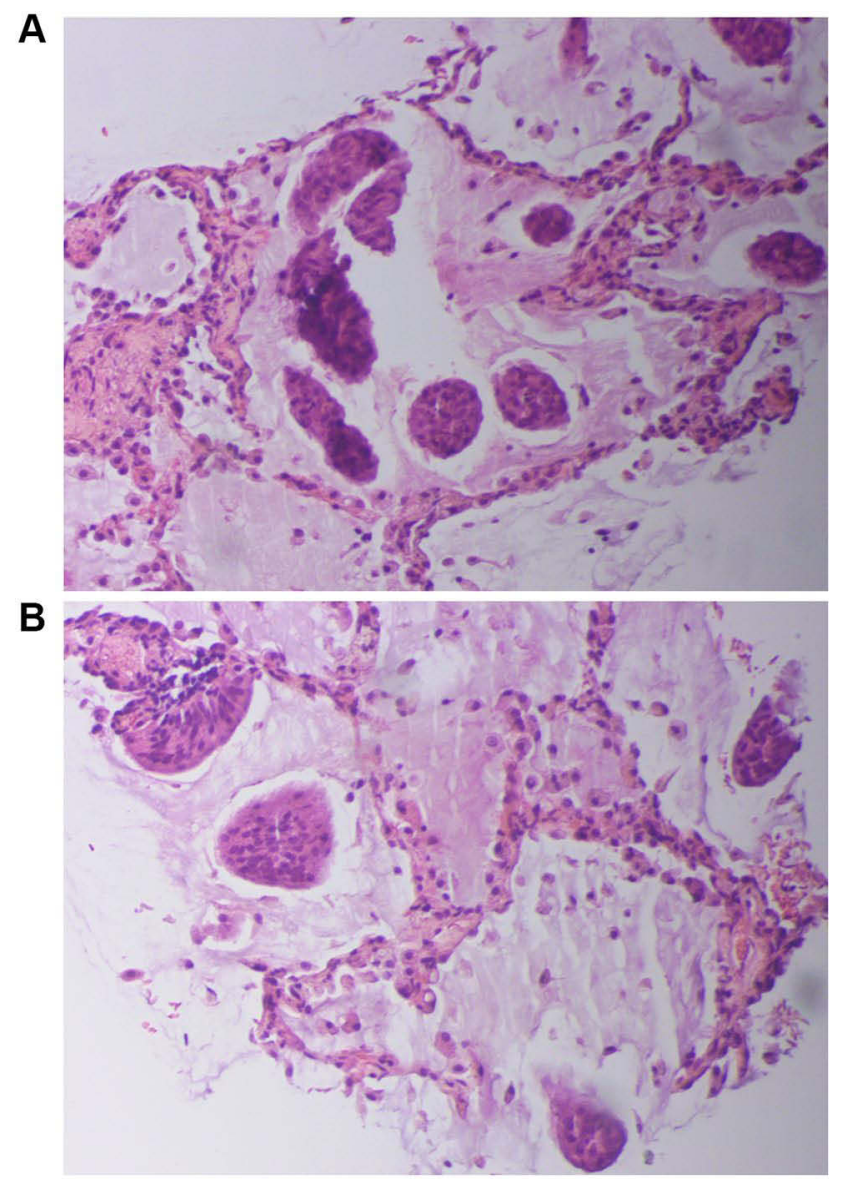

Figure 2 Percutaneous lung biopsy specimen from tumor lesions (A and $\mathbf{B}$, hematoxylin-eosin). Pathology showed a lot of mucus filling the alveolar space.

many fusion partners of ALK, echinoderm microtubuleassociated protein-like 4 (EML4) is the most common fusion partner. The EML4-ALK fusion is formed by the inversion of chromosome 2. This inversion can occur in multiple places on the chromosome, the most common is variant 1. EML4-ALK translocation can result in constitutive ALK kinase activity and represents an oncogenic addiction pathway in lung cancer. The EML4-ALK gene induced tumor formation in nude mice. ${ }^{24,25}$ In NSCLC, ALK rearrangement is more common in adenocarcinoma. These patients have unique clinical characteristics, including a history of never smoking or light smoking, younger age, advanced disease and frequent lymph node metastasis. $^{5}$ The patient was basically consistent with these characteristics in this case. ALK rearrangement is associated with adenocarcinoma with solid or mucus as the main growth pattern. ${ }^{26}$ Furthermore, morphologically, ALK rearrangement is abundant in adenocarcinoma with signet ring cells. ${ }^{27}$ In terms of reality, the ALK-rearranged IMA has acinar and solid growth tumor foci, and is rich in
A

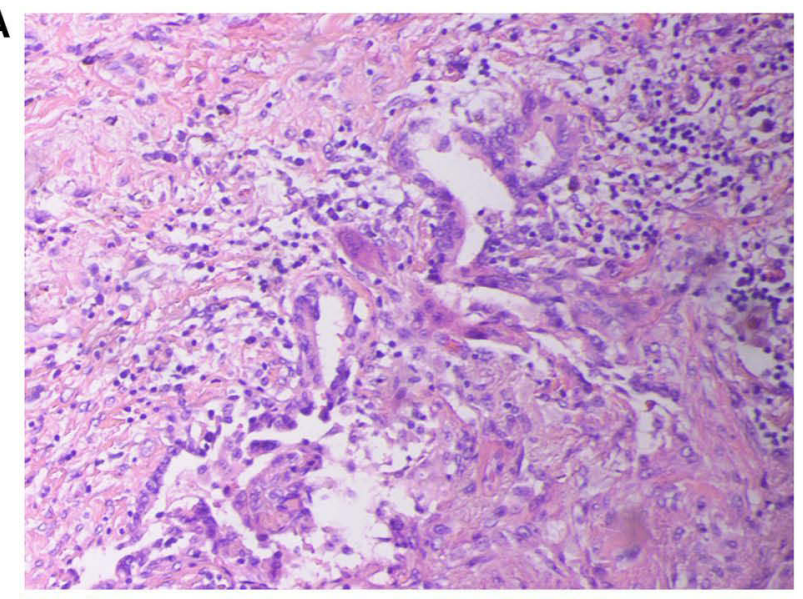

B

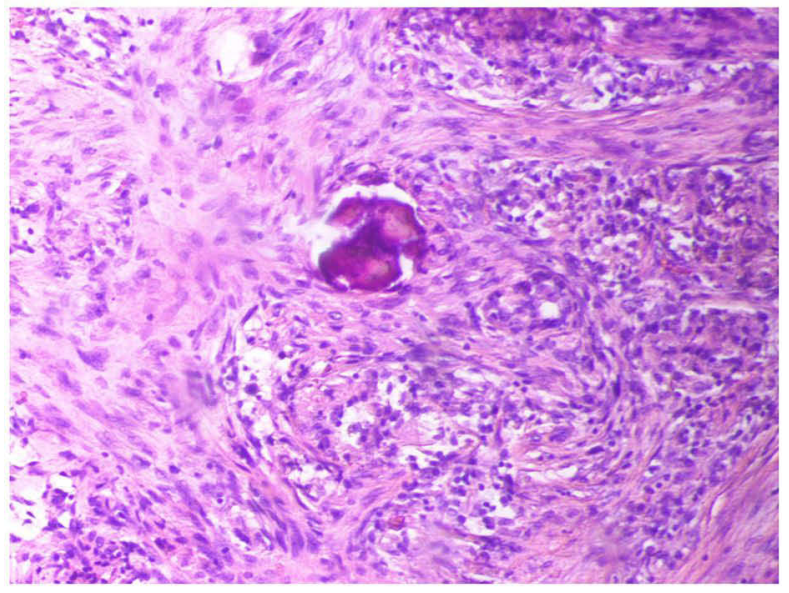

Figure 3 Pathological examination of tissue specimens from thoracoscopic left lobectomy (A and B, hematoxylin-eosin). Pathology showed a small amount of scattered high-grade atypical glands in the interstitium, and fibrous tissue proliferation, foam cell aggregation, lymphocyte infiltration, multinucleated giant cell reaction, necrotic hemicalcium can be seen in the remaining lung tissue.

signet ring cells, which was consistent with the findings of our case.

At present, ALK rearrangement has become the target of new molecular targeted therapy. The use of ALK tyrosine kinase inhibitors (TKI) for genotype targeted therapy has brought a fundamental change in the treatment of patients with NSCLC. So far, 4 ALK TKIs, ceritinib, crizotinib, alectinib and brigatinib, have been approved by the European Medicines Agency (EMA) and/or US Food and Drug Administration (FDA) for targeted therapy of ALK rearranged patients. Alectinib is the secondgeneration ALK TKI. Unlike the first-generation ALK TKI (crizotinib), alectinib is not a substrate of P-glycoprotein (P-gp). It has a higher rate of passing through the blood-brain barrier. The presence of the brain barrier weakens the efficacy of brain metastases, which can significantly reduce the brain metastases of patients and reduce the risk of death. In our case, the 


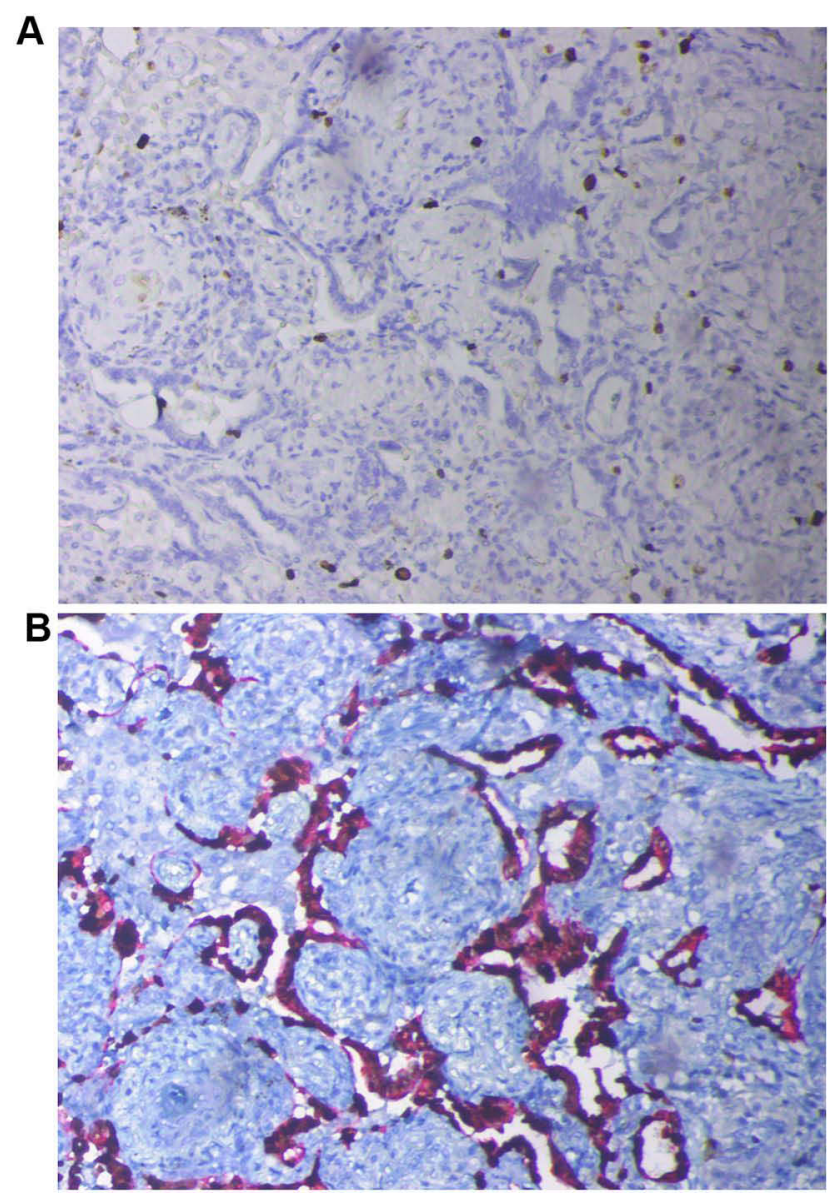

Figure 4 Immunohistochemical staining (20-X) revealed Ki6I positivity (A), cytokeratin 7 (CK7) and thyroid transcription factor-I (TTF-I) positivity (B).

patient achieved both radiologic and pathologic regressions without any adverse events after 3 cycles (84 days) of neoadjuvant alectinib. Obviously, the therapeutic effect of alectinib was amazing.

In a global randomized, Phase 3 ALEX trial, ${ }^{28}$ the progression-free survival rate of the alectinib group was significantly higher than that of the crizotinib group. The ORR of the alectinib group was higher than that of the crizotinib group ( $82.9 \%$ vs $75.5 \%$ ), and there was no statistical difference $(P=0.09)$. Recently, an ALK rearranged IV stage (T2N2M1) lung adenocarcinoma woman was reported. New metastatic lesions were found in the brain and meninges by craniocerebral magnetic resonance imaging (MRI). After alectinib treatment, the clinical symptoms of the patient's brain were significantly improved, and the brain lesions almost disappeared. CNS metastases in ALKpositive NSCLC patients, including pia mater and brain metastases, are increasingly occurring. The J-ALEX study in Japan suggested that compared with crizotinib, the 1-year cumulative incidence of CNS progression of alectinib was significantly reduced $(5.9 \%$ (alectinib) vs $16.8 \%$ (crizotinib)). ${ }^{29}$ Alectinib has been shown to have high intracranial efficacy. Cancer patients may have various adverse reactions (AEs) when receiving treatment, and some need to be discontinued. A lower rate of discontinuation usually indicates that the treatment is safer. In a meta-analysis, the discontinuation rate of alectinib (7\%) was lower than that of ceritinib and crizotinib (8\%), showing the superior safety of alectinib. $^{30}$ The patients we reported have not yet experienced any adverse reactions. The above results show that alectinib has a significant advantage over crizotinib in advanced NSCLC patients with ALK rearrangement. The patient we reported showed favorable results after treatment with alectinib, confirming the superiority of alectinib.

Neoadjuvant treatment of NSCLC with novel therapeutic agents is an emerging field of investigation, aiming to improve the cure rate. Although neoadjuvant therapy may delay surgery and take risks of disease progression, its advantages have already occupied solid foundation. A meta-analysis of NSCLC showed that neoadjuvant chemotherapy could absolutely improve 5-year OS, from $40 \%$ to $45 \% .{ }^{16}$ Previous case reports of patients with locally advanced NSCLC who received EGFR-TKIs in neoadjuvant therapy showed favorable results and induced tumor downstaging. ${ }^{31,32}$ Outstanding activity of alectinib has been demonstrated for the treatment of ALK + NSCLC. However, there are no relevant reports of phase II/III study on the effect of neoadjuvant alectinib treatment. Imanishi et $\mathrm{al}^{33}$ reported a case of IIIA-N2 stage lung adenocarcinoma with ALK rearrangement after 3 months of treatment with alectinib, the tumor was significantly reduced, followed by surgery and complete resection. Pathology showed that in all excised specimens, less than $10 \%$ of tumor cells were alive. This suggests that neoadjuvant alectinib treatment may be effective and safe for patients with lung adenocarcinoma harboring ALK-rearrangements. More recently, Zhang et al reported a clinically successful case of neoadjuvant alectinib in the treatment of stage IIIB ALK + NSCLC. Similar to our report, The tumor was significantly reduced after alectinib induction, and the TNM stage was downstaged from cT3N2M0 IIIB to ypT1aN0M0 IB. ${ }^{18}$ Moreover, another similar clinical success case of alectinib neoadjuvant was reported, a patient with stage IIIA ALK + NSCLC who received alectinib neoadjuvant treatment with a major pathological response, and tumor was downstaged at ypT0N2. Based on this case, Leonetti et $\mathrm{al}^{34}$ further designed a Phase II 
study aimed at assessing alectinib as neoadjuvant treatment in surgically resectable Stage III ALK + NSCLC (ALNEO trial). In the clinic, neoadjuvant chemotherapy represents the standard treatment for patients with resectable locally advanced NSCLC. ALK-TKIs in ALK + NSCLC has better feasibility and safety. In particular, giving alectinib before surgery, as in our case, can induce more extensive tumor shrinkage than standard chemotherapy.

\section{Conclusions}

To our knowledge, this is the first case discussing the treatment of ALK-rearranged IMA of the lung with neoadjuvant alectinib. The patient achieved both radiologic and pathologic regressions after three cycles of oral alectinib, and so far, no adverse reactions have been found. The therapeutic effect of alectinib is remarkable. This case proves that in patients with advanced ALK-rearranged lung adenocarcinoma, neoadjuvant alectinib can achieve favorable therapeutic effects, which can provide a reference for clinical treatment of patients.

\section{Ethics}

This patient provided written informed consent for the publication of the case details and images. And ethical approval for this study was obtained from the meeting of ethics committee of Zhejiang Provincial People's Hospital.

\section{Funding}

This study was funded by the grants from Zhejiang Medical Association (No. 2017ZYC-A47), Zhejiang Provincial Department of Health Project (No. 2021 KY537).

\section{Disclosure}

The authors report no conflicts of interest in this work.

\section{References}

1. Tang ER, Schreiner AM, Pua BB. Advances in lung adenocarcinoma classification: a summary of the new international multidisciplinary classification system (IASLC/ATS/ERS). J Thorac Dis. 2014;6(Suppl 5):S489-501.

2. Siegel R, Ma J, Zou Z, Jemal A. Cancer statistics, 2014. CA Cancer J Clin. 2014;64(1):9-29.

3. Cha YJ, Shim HS. Biology of invasive mucinous adenocarcinoma of the lung. Transl Lung Cancer Res. 2017;6(5):508-512. doi:10.21037/ tlcr.2017.06.10

4. Shim HS, Kenudson M, Zheng Z, et al. Unique genetic and survival characteristics of invasive mucinous adenocarcinoma of the lung. J Thor Oncol. 2015;10(8):1156-1162. doi:10.1097/JTO.0000000000 000579
5. Tian P, Liu Y, Zeng H, et al. Unique molecular features and clinical outcomes in young patients with non-small cell lung cancer harboring ALK fusion genes. J Cancer Res Clin Oncol. 2020;146(4):935-944. doi:10.1007/s00432-019-03116-6

6. Franco R, Rocco G, Marino FZ, et al. Anaplastic lymphoma kinase: a glimmer of hope in lung cancer treatment? Expert Rev Anticancer Ther. 2013;13(4):407-420. doi:10.1586/era.13.18

7. Sabir SR, Yeoh S, Jackson G, Bayliss R. EML4-ALK variants: biological and molecular properties, and the implications for patients. Cancers. 2017;9:9. doi:10.3390/cancers9090118

8. Ou SH, Ahn JS, De Petris L, et al. Alectinib in crizotinib-refractory ALK-rearranged non-small-cell lung cancer: a Phase II global study. J Clin Oncol. 2016;34(7):661-668. doi:10.1200/JCO.2015.63.9443

9. Tamura T, Kiura K, Seto T, et al. Three-year follow-up of an alectinib Phase I/II study in ALK-positive non-small-cell lung cancer: AF-001JP. J Clin Oncol. 2017;35(14):1515-1521. doi:10.1200/ JCO.2016.70.5749

10. Shaw AT, Gandhi L, Gadgeel S, et al. Alectinib in ALK-positive, crizotinib-resistant, non-small-cell lung cancer: a single-group, multicentre, Phase 2 trial. Lancet Oncol. 2016;17(2):234-242. doi:10.1016/S1470-2045(15)00488-X

11. Gadgeel SM, Shaw AT, Govindan R, et al. Pooled analysis of CNS response to alectinib in two studies of pretreated patients with ALK-positive non-small-cell lung cancer. J Clin Oncol. 2016;34 (34):4079-4085. doi:10.1200/JCO.2016.68.4639

12. Ettinger DS, Aisner DL, Wood DE, et al. NCCN guidelines insights: non-small cell lung cancer, Version 5.2018. J Natl Comp Cancer Net. 2018;16(7):807-821. doi:10.6004/jnccn.2018.0062

13. Camidge DR, Dziadziuszko R, Peters S, et al. Updated efficacy and safety data and impact of the EML4-ALK fusion variant on the efficacy of alectinib in untreated alk-positive advanced non-small cell lung cancer in the global Phase III ALEX study. J Thor Oncol. 2019;14(7):1233-1243. doi:10.1016/j.jtho.2019.03.007

14. Gadgeel S, Peters S, Mok T, et al. Alectinib versus crizotinib in treatment-naive anaplastic lymphoma kinase-positive (ALK+) non-small-cell lung cancer: CNS efficacy results from the ALEX study. Ann Oncol. 2018;29(11):2214-2222. doi:10.1093/annonc/ mdy405

15. Montemurro F, Nuzzolese I, Ponzone R. Neoadjuvant or adjuvant chemotherapy in early breast cancer? Expert Opin Pharmacother. 2020;21(9):1071-1082. doi:10.1080/14656566.2020.1746273

16. Group NM. Preoperative chemotherapy for non-small-cell lung cancer: a systematic review and meta-analysis of individual participant data. Lancet (London, England). 2014;383(9928):1561-1571. doi:10.1016/S0140-6736(13)62159-5

17. Leonetti A, Minari R, Boni L, et al. Phase II, open-label, single-arm, multicenter study to assess the activity and safety of alectinib as neoadjuvant treatment in surgically resectable stage III ALK-positive NSCLC: ALNEO trial. Clin Lung Cancer. 2021;22 (5):473-477.

18. Zhang C, Yan LX, Jiang BY, Wu YL, Zhong WZ. Feasibility and safety of neoadjuvant alectinib in a patient with ALK-positive locally advanced NSCLC. J Thor Oncol. 2020;15(6):e95-e99. doi:10.1016/j. jtho.2019.12.133

19. Cai D, Li H, Wang R, et al. Comparison of clinical features, molecular alterations, and prognosis in morphological subgroups of lung invasive mucinous adenocarcinoma. Onco Targets Ther. 2014;7:2127-2132.

20. Shimizu K, Okita R, Saisho S, Maeda A, Nojima Y, Nakata M. Clinicopathological and immunohistochemical features of lung invasive mucinous adenocarcinoma based on computed tomography findings. Onco Targets Ther. 2017;10:153-163. doi:10.2147/OTT. S121059

21. Sun F, Wang P, Zheng Y, et al. Diagnosis, clinicopathological characteristics and prognosis of pulmonary mucinous adenocarcinoma. Oncol Lett. 2018;15(1):489-494. 
22. Kim H, Jang SJ, Chung DH, et al. A comprehensive comparative analysis of the histomorphological features of ALK-rearranged lung adenocarcinoma based on driver oncogene mutations: frequent expression of epithelial-mesenchymal transition markers than other genotype. PLoS One. 2013;8(10):e76999. doi:10.1371/journal.pone. 0076999

23. Chiarle R, Voena C, Ambrogio C, Piva R, Inghirami G. The anaplastic lymphoma kinase in the pathogenesis of cancer. Nat Rev Cancer. 2008;8(1):11-23. doi:10.1038/nrc2291

24. Soda M, Choi YL, Enomoto M, et al. Identification of the transforming EML4-ALK fusion gene in non-small-cell lung cancer. Nature. 2007;448(7153):561-566. doi:10.1038/nature05945

25. Hallberg B, Palmer RH. Mechanistic insight into ALK receptor tyrosine kinase in human cancer biology. Nat Rev Cancer. 2013;13 (10):685-700. doi:10.1038/nrc3580

26. Shaw AT, Yeap BY, Mino-Kenudson M, et al. Clinical features and outcome of patients with non-small-cell lung cancer who harbor EML4-ALK. J Clin Oncol. 2009;27(26):4247-4253. doi:10.1200/ JCO.2009.22.6993

27. Possidente L, Landriscina M, Patitucci G, Borgia L, Lalinga V, Vita G. ALK rearrangement in specific subtypes of lung adenocarcinoma: immunophenotypic and morphological features. Med Oncol. 2017;34(5):76. doi:10.1007/s12032-017-0936-Z

28. Peters S, Camidge DR, Shaw AT, et al. Alectinib versus crizotinib in untreated ALK-positive non-small-cell lung cancer. $N$ Engl J Med. 2017;377(9):829-838. doi:10.1056/NEJMoa1704795
29. Nishio M, Nakagawa K, Mitsudomi T, et al. Analysis of central nervous system efficacy in the J-ALEX study of alectinib versus crizotinib in ALK-positive non-small-cell lung cancer. Lung Cancer (Amsterdam, Netherlands). 2018;121:37-40. doi:10.1016/j.lungcan. 2018.04.015

30. Fan J, Fong T, Xia Z, Zhang J, Luo P. The efficacy and safety of ALK inhibitors in the treatment of ALK-positive non-small cell lung cancer: a network meta-analysis. Cancer Med. 2018;7(10):4993-5005. doi:10.1002/cam4.1768

31. Kappers I, Klomp HM, Burgers JA, Van Zandwijk N, Haas RL, van Pel R. Neoadjuvant (induction) erlotinib response in stage IIIA non-small-cell lung cancer. J Clin Oncol. 2008;26(25):4205-4207. doi:10.1200/JCO.2008.16.3709

32. Takamochi K, Suzuki K, Sugimura H, et al. Surgical resection after gefitinib treatment in patients with lung adenocarcinoma harboring epidermal growth factor receptor gene mutation. Lung Cancer (Amsterdam, Netherlands). 2007;58(1):149-155. doi:10.1016/j.lungcan.2007.04.016

33. Imanishi N, Yoneda K, Taira A, et al. Major pathologic response to alectinib in ALK-rearranged adenocarcinoma of the lung. Surg Case Rep. 2018;4(1):19. doi:10.1186/s40792-018-0430-7

34. Leonetti A, Minari R, Boni L, et al. Phase II, open-label, single-arm, multicenter study to assess the activity and safety of alectinib as neoadjuvant treatment in surgically resectable Stage III ALK-positive NSCLC: ALNEO trial. Clin Lung Cancer. 2021;22(5):473-477.

\section{Publish your work in this journal}

OncoTargets and Therapy is an international, peer-reviewed, open access journal focusing on the pathological basis of all cancers, potential targets for therapy and treatment protocols employed to improve the management of cancer patients. The journal also focuses on the impact of management programs and new therapeutic agents and protocols on patient perspectives such as quality of life, adherence and satisfaction. The manuscript management system is completely online and includes a very quick and fair peer-review system, which is all easy to use. Visit http://www.dovepress.com/ testimonials.php to read real quotes from published authors. 